Article

\title{
Modeling Electric Discharges with Entropy Production Rate Principles
}

\section{Thomas Christen}

ABB Schweiz AG, Corporate Research, Segelhofstrasse 1, CH-5405 Baden-Dättwil, Switzerland; E-Mail: thomas.christen@ch.abb.com

Received: 29 October 2009 / Accepted: 01 December 2009 / Published: 8 December 2009

\begin{abstract}
Under which circumstances are variational principles based on entropy production rate useful tools for modeling steady states of electric (gas) discharge systems far from equilibrium? It is first shown how various different approaches, as Steenbeck's minimum voltage and Prigogine's minimum entropy production rate principles are related to the maximum entropy production rate principle (MEPP). Secondly, three typical examples are discussed, which provide a certain insight in the structure of the models that are candidates for MEPP application. It is then thirdly argued that MEPP, although not being an exact physical law, may provide reasonable model parameter estimates, provided the constraints contain the relevant (nonlinear) physical effects and the parameters to be determined are related to disregarded weak constraints that affect mainly global entropy production. Finally, it is additionally conjectured that a further reason for the success of MEPP in certain far from equilibrium systems might be based on a hidden linearity of the underlying kinetic equation(s).
\end{abstract}

Keywords: entropy production rate principles; Steenbeck principle; Prigogine principle; electric arc; charge injection

\section{Introduction}

Electric (gas) discharges appear in various natural phenomena like lightning, St. Elmo's fire, and spark discharges. Moreover, electric discharges are exploited in various technical devices as lamps, circuit breakers, and plasma torches, to mention a few. Despite of the seniority of the "science of discharges" and the today's increased computational power, the complexity of the involved physical phenomena like radiative transfer, plasma flow (sometimes supersonic and/or turbulent), electrical contact physics, etc., 
still resists a rigorous and general theoretical treatment. Almost eighty years ago, Max Steenbeck wrote in a seminal scientific note [1], that at fixed current, the heat power, and thus the voltage drop between the electrodes, is minimized in a real gas discharge. Since then, Steenbeck's principle has been often used by engineers as a "quick and dirty" approach for modeling electric gas discharge applications [2]. According to Peters [3], it can be traced back to Prigogine's principle of minimum entropy production rate [4]. Results obtained from these principles often reproduce experimental observations astonishingly well. This fact requires an explanation, because Prigogine's principle is restricted to linear deviations from thermodynamic equilibrium (for which we will use the terms "weak nonequilibrium" and "near equilibrium"). However, most types of gas discharges exhibit non-ohmic current-voltage relations and are thus beyond linear response (i.e., "far from equilibrium").

This article addresses aspects of the pragmatic question, under which circumstances entropy production principles can be helpful for modeling electric discharge phenomena far from equilibrium. "Modeling" will here be associated with relatively simple phenomenological models that provide, for the physical quantities of interest, quantitative predictions, which are probably not exact but serve as estimates with sufficient accuracy for practical purposes. Exhaustive review articles on variational principles for the entropy production rate and similar principles can be found in [5-7]. We will first show that Steenbeck's and Prigogine's principles are consequences of the maximum entropy production rate principle (MEPP) [8]. We then illustrate their application by three examples, in order to obtain intuition needed for the somewhat vague (as is in the nature of it) conceptual discussion conducted at the end of this paper.

\section{Steenbeck's Principle from MEPP}

Figure 1 sketches the type of system that will be considered throughout this paper [8]. It is isolated and consists of a subsystem $\Omega_{t o t}$, bounded by the dashed curve, and a much larger part, $\Sigma$, that acts as a thermal equilibrium heat bath with constant temperature $T_{a m b}$ and that is at electrical ground. The electrical coupling between $\Sigma$ and $\Omega_{t o t}$ is purely capacitive, and the total charge in each is zero. The boundary $\partial \Omega_{t o t}$ of $\Omega_{t o t}$ is chosen to be at ground potential and at ambient temperature $T_{a m b}$.

An initial nonequilibrium state is then prepared by charging a battery with energy $W_{0}$, and keeping first everything else in $\Omega_{t o t}$ at equilibrium ( $T=T_{a m b}$ everywhere and vanishing current $I=0$ ). The battery is assumed to be ideal, i.e., it provides a constant voltage $U$ as long as electric energy is stored, and has zero voltage if it is empty. Leakage losses will be neglected, and series discharge losses in the battery can be included in the general ohmic resistor $R_{0}$ connected in series to the (fully dissipative) subsystem $\Omega$. The latter, which is in the main focus, is to be modeled later.

When a discharge starts, a constant current $I$ develops. This steady state will have a spatial temperature distribution $T(\mathbf{r}), \mathbf{r} \in \Omega_{t o t}$. Its discharge duration $t_{d}$, defined by the battery being emptied, is assumed to be much longer than the initial and final transient states when the steady state forms and decays, respectively. The final equilibrium state is characterized by a fully discharged battery and again a temperature $T_{a m b}$ everywhere. Equality of initial and final temperature is ensured by $\Sigma$ being a thermodynamic reservoir, i.e., $W_{0}$ being much smaller than the total thermal energy content of $\Sigma$. The initially stored potential energy $W_{0}$ is eventually fully dissipated into heat. The entropy difference of 
final and initial states is then $W_{0} / T_{a m b}$. Since the short transient phases can be neglected, the entropy production rate $W_{0} / t_{d} T_{a m b}=P / T_{a m b}$ is constant, with $P$ being the steady state power in $\Omega_{t o t}$.

Figure 1. The isolated nonequilibrium system, consisting of a part $\Omega_{t o t}$ with variable temperature $T(\mathbf{r})$ inside the dashed boundary (containing a battery with voltage $U$, a constant resistor $R_{0}$, an electrical sub-system $\Omega$ ), and the heat reservoir $\Sigma$ at temperature $T_{a m b}$ and at ground potential.

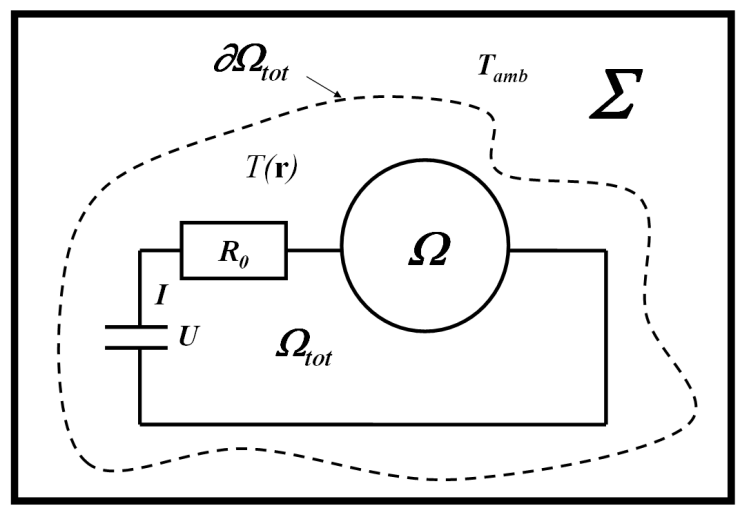

For simplicity, we suppose that the "device" $\Omega$ is only electrically and thermally coupled to its environment, but does not exchange mass or momentum. This device is described by a phenomenological model $U_{\Omega}(I, z)$ for the current voltage relation, containing a number of model parameters $\mathbf{z}=\left(z_{1}, z_{2}, \ldots, z_{N}\right)$ with yet unknown values. Complete information would be given by $N$ appropriate equations from which $\mathbf{z}$ can be determined; the problem would then be solved. We assume, however, that some information on $\Omega$, is missing, i.e., only part of these equations are known. The goal will then be to determine the values of $\mathbf{z}$ with MEPP, using the given equations as constraints. MEPP states that the total system restores equilibrium by maximizing the entropy production rate $\dot{S}$ (of the quasi-steady state) subject to the given constraints. One of the constraints is total power balance

$$
P=U I
$$

where $U I$ is the power delivered by the battery, and

$$
P=R_{0} I^{2}+P_{\Omega}
$$

is the total Joule heat production. $P_{\Omega}=U_{\Omega} I$ is the heat production of subsystem $\Omega$. As shown above, the total heat production, $P$, in $\Omega_{t o t}$ is related to the total entropy production rate $\dot{S}$ by [3]

$$
\dot{S}(\mathbf{z})=\frac{P(\mathbf{z})}{T_{a m b}}
$$

MEPP is here obviously equivalent to maximum total power $P(\mathbf{z})$. Note that $U$ is a fixed parameter but $I$ varies with $\mathbf{z}$. Besides the total power, only the ambient temperature appears explicitly in Equation 3, although in $\Omega_{t o t}$ a non-constant temperature distribution, $T(\mathbf{r})$, generally exists. For instance, if $T_{0}$ and $T_{\Omega}$ are the temperatures of the resistor $R_{0}$ and $\Omega$, respectively, $\dot{S}$ is not equal to 
$R_{0} I^{2} / T_{0}+P_{\Omega} / T_{\Omega}$, which neglects a part of the entropy production due to heat flow. It is thus crucial to choose the surface $\partial \Omega_{t o t}$ at ambient temperature. Because $R_{0}$ (cf. Figure 1) contributes to dissipation, it is inappropriate to apply Equation 3 to the system $\Omega$ only [8]: the heat production $R_{0} I^{2}$ must not be neglected! But this is exactly what is often implicitly done when Prigogine's principle of minimum entropy production is used in discharge applications. Prigogine's principle assumes fixed current, which implies that the main entropy production appears in $R_{0}$ outside $\Omega$ ! Indeed, imposed $I$ means $R_{0} \rightarrow \infty$ and $U \rightarrow \infty$ with finite ratio $U / R_{0}=I$. Prigogine's principle for $\Omega$ is thus only valid in leading order $I^{2}$ in Equation 3, where terms of order $T-T_{a m b}$ can be neglected. Of course, Prigogine's principle works if one minimizes $P_{\Omega} / T_{a m b}$ at constant $I$, as is usually done. But this is misleading since $P_{\Omega} / T_{a m b}$ has no physical meaning in general, because its derivation requires a boundary, which encloses the dissipating system $\Omega$, excludes $R_{0}$, and is at constant temperature $T_{a m b}$ (cf. [3]) - and this boundary does not exist in general.

MEPP refers to maximization of $\dot{S}(\mathbf{z})=U I / T_{a m b}$ at fixed $U$. This implies maximum current $I$ under variation of $\mathbf{z}$. Without any qualification, we assume that the given information (i.e., the constraints) has been used to eliminate as many parameters as possible, and $\mathrm{z}$ contains the remaining unknowns. Using $d S=U d I / T_{a m b}$ and the mesh rule, $U=R_{0} I+U_{\Omega}(I, \mathbf{z})$, one obtains then

$$
T_{a m b} \nabla_{\mathbf{z}} \dot{S}=\frac{-U}{R_{0}+\partial U_{\Omega} / \partial I} \nabla_{\mathbf{z}} U_{\Omega}
$$

with $\nabla_{\mathbf{z}}=\left(\partial / \partial z_{1}, \partial / \partial z_{2}, \ldots\right)$. The maximum entropy production (MEP) state is a local optimum if $\nabla_{\mathbf{z}} \dot{S}=0$, i.e., $\nabla_{\mathbf{z}} U_{\Omega}=0$. Let the solution be denoted by $\mathbf{z}_{0}$. In order that $\dot{S}$ is a maximum, the second derivative

$$
T_{a m b}\left(\frac{\partial^{2} \dot{S}}{\partial z_{j} \partial z_{k}}\right)_{\mathbf{z}_{0}}=\frac{-U}{R_{0}+\left(\partial U_{\Omega} / \partial I\right)_{\mathbf{z}_{0}}}\left(\frac{\partial^{2} U_{\Omega}}{\partial z_{j} \partial z_{k}}\right)_{\mathbf{z}_{0}}
$$

must be negative (semi-) definite. There are two different cases, depending on whether the denominator of the prefactor in Equation 5, i.e., the differential resistance, $d U_{\Omega} / d I+R_{0}$, of the total system is positive or negative. Note that in Equation $5\left(\partial U_{\Omega} / \partial I\right)_{\mathbf{z}_{0}}=d U_{\Omega} / d I$ because $\left(\nabla_{\mathbf{z}} U_{\Omega}\right)_{\mathbf{z}_{0}}=0$.

In the former case, MEPP requires that $\partial^{2} U_{\Omega} / \partial z_{j} \partial z_{k}$ must be positive (semi-)definite. Hence the voltage is a minimum of $U_{\Omega}(I, \mathbf{z})$ as a function of $\mathbf{z}$ at constant $I$, as stated by Steenbeck's principle. This is equivalent to saying that the system $\Omega$ is minimizing its resistance $R_{\Omega}(\mathbf{z})=U_{\Omega} / I$. For negative differential resistance of $\Omega, d U_{\Omega} / d I<0$, the Steenbeck voltage minimum is a MEP state only in presence of a sufficiently large resistor, i.e., if $R_{0}+d U_{\Omega} / d I>0$. It is a well-known fact that (macroscopic) electric systems with negative differential conductance must be stabilized with an appropriately large series resistance [9].

On the other hand, MEPP is also satisfied for a state that maximizes voltage at fixed $I$ and has negative $R_{0}+d U_{\Omega} / d I$. Usually, a finite voltage maximum does not exist, and this state is for most cases unphysical and characterizes just the extinction of a gas discharge, or its instability and cross-over to a different discharge state.

\section{Applications}

There are roughly three classes of questions addressed by modeling electric discharge structures: (1) What happens at boundaries and electrodes? (2) What are the values of the variables characterizing 
the discharge structure? (3) What is the behavior of the discharge structure as a whole in its specific environment? In the following, we illustrate with simple textbook examples how MEPP is answering these questions. In particular, we discuss (1) unipolar charge injection from an electrode, (2) a simple column model for an electric arc, and (3) arc root attachment at the anode in a plasma torch. These examples are not new, but they may give hints for which types of model MEPP can be useful.

\subsection{Charge Injection from an Electric Contact}

Consider a medium, without intrinsic charge carriers, between two parallel metal electrodes separated by a distance $L$. Electric conduction can only appear via carriers injected from an electrode. In order to prevent irrelevant sign manipulations, we will assume positive polarity of the injected carriers, although in most cases electrons, or negative ions formed via electron attachment, are concerned.

For unipolar injection from an electrode at potential $V$ (while the counter electrode is grounded), the injected carrier density $n$ gives rise to the space charge density $\rho=e n$ ( $e$ is the elementary charge). Due to scattering with the (sufficiently dense) medium, the carriers have a mobility $\mu$. The injecting electrode is considered as a charge reservoir that is able to provide, above a critical field value $E_{c}$, charge carriers without any significant resistance; below $E_{c}$ injection is absent and the field distribution is purely capacitive. The specific charge injection mechanism (thermal emission, surface roughness induced Corona, etc.) is irrelevant here. If we consider plane geometry (i.e., 1d), the current density, $j=\mu \rho E$, is spatially constant. The electric field $E$ obeys the Poisson equation, $\epsilon d E / d x=\rho$, where $x$ is the $1 \mathrm{~d}$-coordinate and $\epsilon$ is the (constant) permittivity. Combination of these equations gives $2 j=\mu \epsilon d\left(E^{2}\right) / d x$, and spatial integration yields

$$
E(x)=\sqrt{E_{0}^{2}+\frac{2 j}{\mu \epsilon} x}
$$

The integration constant $E_{0}$ is the unknown parameter $z$, which will be determined with MEPP. The constraint reads $E(0)=E_{0} \geq E_{c}$ for $I>0$. Hence, for $U_{\Omega}<E_{c} L$, it holds $E(x)=U_{\Omega} / L$ and $I=0$, while for larger $U_{\Omega}$, Equation 6 must be considered. Spatial integration from $x=0$ to $x=L$ gives

$$
U_{\Omega}\left(I, E_{0}\right)=\frac{A \mu \epsilon}{3 I}\left(\left(E_{0}^{2}+\frac{2 I L}{A \mu \epsilon}\right)^{3 / 2}-\left(E_{0}^{2}\right)^{3 / 2}\right)
$$

where $I=j A$ with $A$ being the cross section area. Applying Steenbeck's principle, the voltage has the constraint minimum at fixed $I$ for $E_{0}=E_{c}$. The field and space charge distributions are sketched in Figure 2. From MEPP one thus finds that the electric field at a contact that "injects" bulk charge above a field $E_{c}$ is suppressed to the field value $E_{c}$ [10]. This effect works also in more complicated geometries, acts to reduce field-enhancements, and can be important in applications, like Corona stabilization [11]. Furthermore, for $E_{c}=0$, the contacts are called "ohmic contacts" [12], where a space charge limited current-voltage characteristics $j=9 \mu \epsilon U_{\Omega}^{2} / 8 L^{3}$ holds. Note that the optimum occurs here at the boundary of the constraint region $E(0) \geq E_{c}$. 
Figure 2. Space charge influenced electric field (solid), space charge density (dashed; $\rho(0)=j / \mu E_{0}$ is the space charge at the electrode $x=0$ ), and capacitive field (dotted).

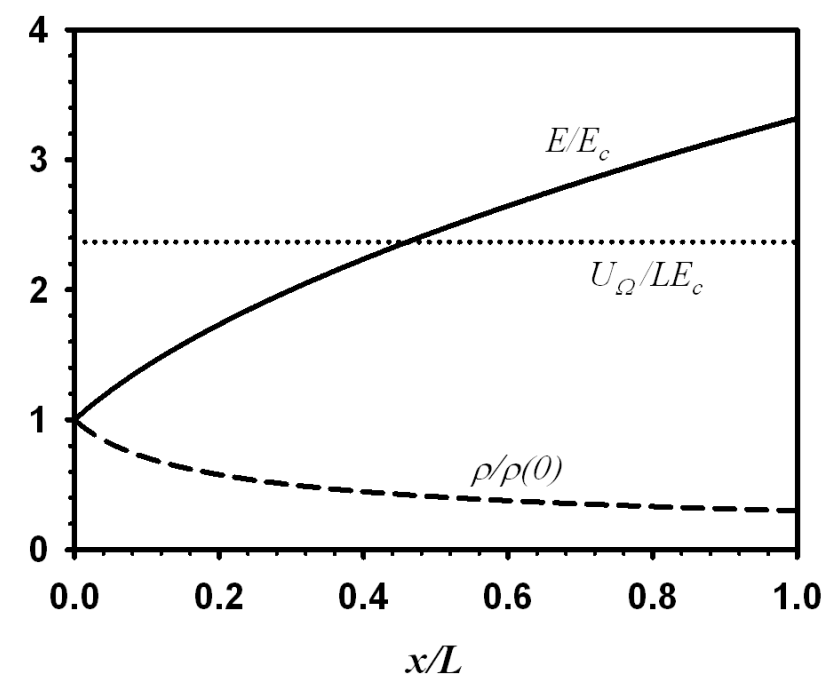

\subsection{Electric Arcs}

High current electric arc columns are among the most frequent applications of Steenbeck's principle [2,9]. We disregard electrode fall voltages and magnetic effects, and assume a cylindrical arc column with radius $r$ and constant core temperature $T$ (see Figure 3). The constraint for $\mathbf{z}=(r, T)$ is heat balance

$$
P_{A r c}(\mathbf{z})=K_{A r c}(\mathbf{z})
$$

were $P_{A r c}=I^{2} / \pi r^{2} \sigma(T)$ is the Joule power (per length) with temperature dependent electric conductivity $\sigma(T)$. The cooling power (per length) of the arc core is modeled by $K_{A r c}=C r^{n} T^{m}$, with constant parameters $C, m$, and $n$. We assume $n=2$ for bulk radiation of an optically thin arc, in contrast to blackbody radiation with $n=1$ for optically thick arcs.

By elimination of $r$ with the help of Equation 8, the MEP state is obtained from minimization of

$$
P_{A r c}(T)=P_{N} \sqrt{\frac{T^{m}}{\sigma(T)}}
$$

The resulting arc temperature is obviously independent of $I$ that is contained only in the prefactor $P_{N}=I \sqrt{C / \pi}$. In contrast to [8] (where the oversimplified arc model discussion contains some inconsistencies), we consider here more realistic conductivity data of air at 1 bar (see Figure 3a)) in order to model a free arc. For $m=3,4,5$, the quantity $P_{A r c} / P_{N}$ as a function of $T$ is shown in Figure 3b. One observes that the MEP temperature $T_{A r c}$ (the minimum of $P_{A r c}(T)$ ) is around $8,000-10,000 \mathrm{~K}$. The dependence of $T_{A r c}$ on $m$ is weak in this range of $m$ values, implying a certain robustness of the arc temperature value. Although this very crude model cannot be expected to give exact results, the order of magnitude of the temperature is quite reasonable for this type of free arc. 
Figure 3. (a) Air conductivity at 1 bar. Inset: power balance for a cylindrical electric arc (Joule power $P$, cooling power $K$, current $I$, radius $r_{A r c}$, and arc core temperature $T_{A r c}$ ). (b) Power as a function of $T$; the minimum provides the arc temperature associated with the MEPP state.
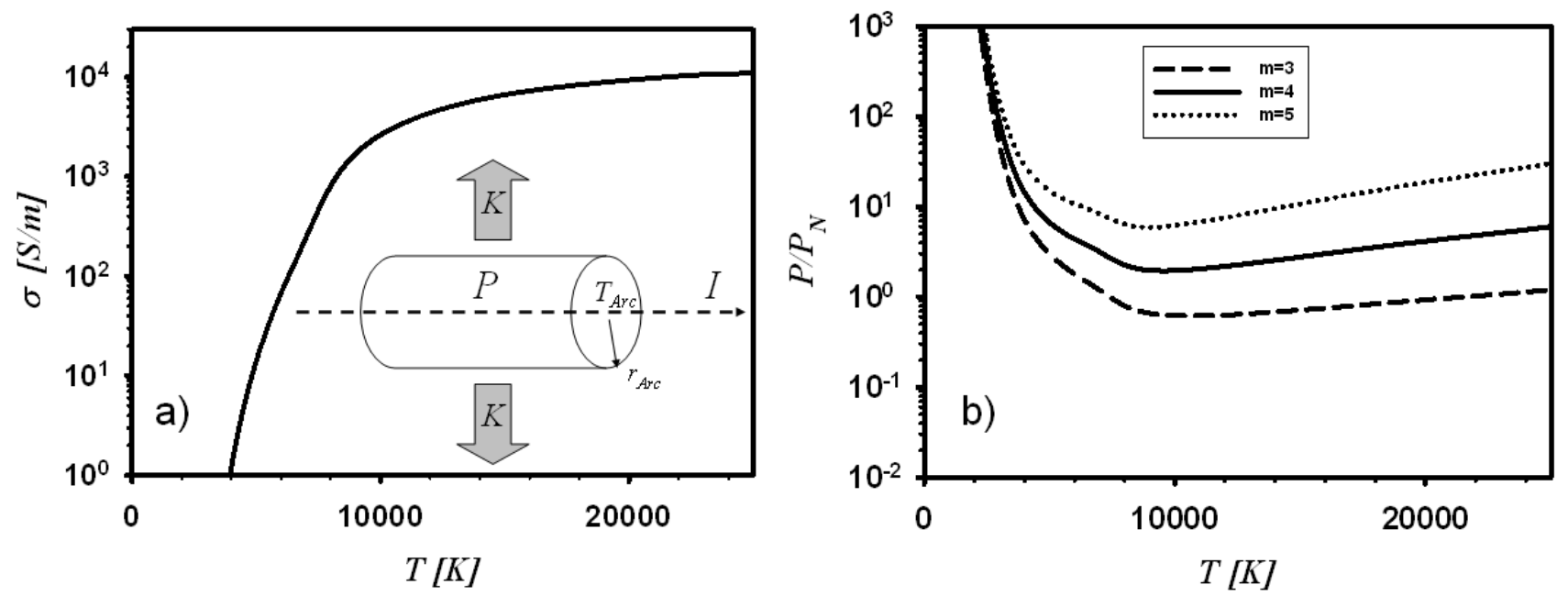

\subsection{Anode Arc Root Position in Plasma Torches}

Plasma torches are widely used for plasma spraying, cutting and welding. A sketch of a plasma torch is shown in Figure 4. The working gas enters from the left side at the cathode into the cylindrical tube, is decomposed into a plasma by the action of an arc, and leads to a plasma jet that leaves at the right side. The arc column extends from the cathode into axial direction (cathode column) and bends, at a certain axial distance $z_{A}$, towards the anode surface (anode column). A main problem is the prediction of the location $z_{A}$ of the arc root. The anode arc root intrinsically breaks the cylindrical symmetry of the system. As a consequence, reliable simulations of plasma torches should be three-dimensional, which makes the simulation problem difficult and cumbersome.

One may, alternatively, apply MEPP, e.g., in the form of Steenbeck's principle by minimizing the voltage drop along the arc $[13,14]$. We assume that arc column properties, for instance its electric field $E$ in a given local arc state, are known. The existence of a voltage minimum as a function of $z_{A}$ (i.e., a MEP state) can then be easily understood. The total voltage consists of the electrode fall voltages (which are assumed to be independent of $z_{A}$ ), the voltage $V_{C}$ along the cathode column, and the voltage $V_{A}$ along the anode column. It is clear that roughly $V_{C}\left(z_{A}\right) \approx E z_{A}$ is proportional to $z_{A}$. Furthermore, the smaller $z_{A}$, the colder the gas that blows the radially directed anode column, and thus the higher the anode column resistance. For constant current, this implies that $V_{A}\left(z_{A}\right)$ is a decreasing function. Reference [14] proposes for illustration $V_{A}=V_{0}\left(1-\exp \left(-c_{0} / z_{A}\right)\right)$, where $V_{0}$ and $c_{0}$ are functions of 
the arc state and the gas flow properties. Because these are approximately constant upon variation of $z_{A}$, minimizing $V_{C}+V_{A}$ gives

$$
z_{A}=\sqrt{\frac{V_{0} c_{0}}{E}}
$$

In [14], the Steenbeck principle for the arc root position was applied in combination with three-dimensional numerical simulations and led to predictions for the arc root position, which are consistent with experimental observations. Another possible application of MEPP to torch arcs might be to model electrode spots with the Steenbeck principle in order to obtain anode or even cathode spot voltage drop (cf. [2]).

Figure 4. Plasma arc torch as described in the text.

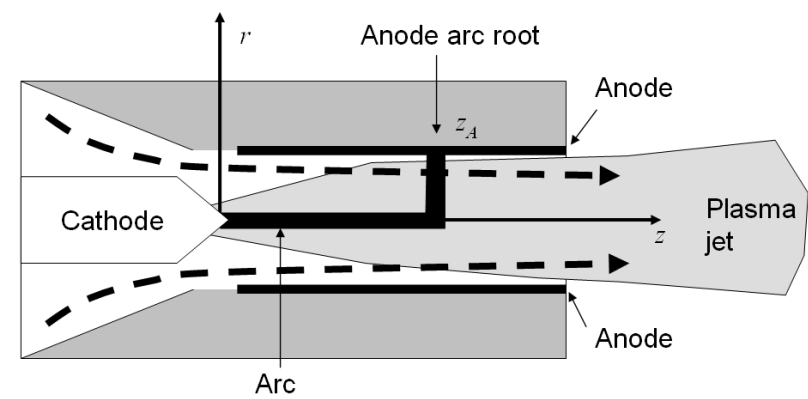

These three examples show how values for a priori unknown model parameters can be obtained with Steenbeck's principle and thus with MEPP. All of them refer to systems with nonlinear current voltage behavior (the arc can even have negative differential resistance, depending on the value of $n$ in the example above). Note that the nonlinearities enter the model via prior information contained in the constraints, and are in general not derived from MEPP. All examples lead to reasonable results, but the parameter values are not "exact" values. They may be interpreted as estimates.

\section{Implications for the Applicability of MEPP}

Entropy production rate principles for steady states are generally valid only near equilibrium. Let us first comment on MEPP for this case. According to Section 2., the maximum and minimum entropy production rate (or power dissipation) principles for steady states are equivalent. Which one applies depends on the convexity properties of the optimization problem as is illustrated in an Appendix. In the isolated system with fixed total voltage, the current $I$ is the natural quantity that dynamically adjusts for equilibration, and MEPP applies. Minimization of $\dot{S}$ is valid if $I$ is fixed, and $U_{\Omega}$ will adjust accordingly. Both cases can be summarized by "minimization of the total resistance". This result is in line with [15], where the deeper reason for the type of optimum is claimed to lie in the time reversal behavior of the basic variables. As these authors have shown, this discussion clarifies and rectifies one of Landauer's arguments [16] against Prigogine's principle.

A proof for the general validity of a variational principle beyond linear response does not exist [17], despite various attempts (see references cited in [6]). One must also not withhold severe 
and sometimes justified criticism in the literature against entropy production principles by various authors (see, e.g., [16-19]). However, criticism usually disregards the existence of modeling problems with a lack of information that often appears in practice. The reason for such ignorance can be manifold, but this does not play a role here. Applying entropy production principles can then be helpful even for finding apparently far-from-equilibrium states. Examples have been presented in the previous section, and there are more of them [6]. On the other hand, it is clearly easy for a critic to "invalidate" the MEPP principle, either by applying it in an inappropriate way or by disregarding relevant constraints, and to produce seemingly disproving results.

The task of MEPP is to determine the values of unknown model parameters, $z$. The believe is that the MEP state gives a "best" or "most unbiased" estimate on the basis of the available information, which is contained in the model (the choice of $\mathbf{z}$, the expression for $\dot{S}(\mathbf{z})$, and the constraints, like balance equations etc.). This viewpoint has been introduced by Jaynes [20] in his framework of predictive statistical mechanics, and was further discussed by Jones [21] and Dewar (see Dewar's chapter in [7], p. 41). Because there is no proof at hand, one is led to the following, more pragmatic, questions: For which cases can MEPP serve as a useful tool? How good is a solution obtained with the help of MEPP?

An often heard statement, tempting for using MEPP, is that an irreversible nonequilibrium system tries to increase its entropy as fast as it can, where "as it can" refers to the constraints. Although not strictly correct in general, it sketches a typical trend behavior of irreversible systems [20]. Thus, far from equilibrium, MEPP can probably be only justified by the tautology, that it is a model-parameter estimate based on the assumption of MEP. The results are the more accurate, the higher the quality of the information contained in the model is. An illuminating example is given by Rebhan [22], who compares the exact shock front solution in an ideal gas with MEPP solutions for all cases, where one of the three hydrodynamic balance equations for mass, momentum, and energy, is disregarded.

Let us illustrate what "quality of information" means in practice for the examples discussed in the previous section. Assuming local thermal equilibrium and a single component fluid description of the arc plasma with known conductivity, the complete information consists in the three hydrodynamic balance equations (together with all initial conditions, boundary conditions, and Ohm's law). For the arc model above, we used information on the structure of the arc, i.e., a hot cylinder, and kept only energy balance. Using mass or momentum balance instead, would lead to not nearly as reasonable results. Why? Because the physics of free (high current) arcs is mainly governed by power balance, due to their significant Joule heat production in the hot plasma column. Energy balance controls the much weaker modes (degrees of freedom) associated with mass and momentum distributions. For example, the mass distribution follows more or less the needs of the power balance: when somewhat more mass is required to satisfy energy balance, it will be sucked into the arc core from the surrounding without significant resistance. Only in the case of a concrete physical mechanism for such resistance, like mass production due to material ablation by radiation of an arc constricted in a tube [23], or a strong, externally forced mass flow, as in certain types of gas circuit breakers [24], mass balance may become as predominant as energy balance.

Similarly, the charge injected in the first example is a "weak" mode, because the electrode is able to provide whatever charge is needed. For instance, ohmic contact behavior is exactly reproduced by MEPP in this case. But when the steady state net current becomes of the order of the injection current of the 
contact, contact physics starts to play a role. In that case, relevant information (i.e., constraints associated with contact physics) is missing in the used model, with the consequence that MEPP becomes worse and worse the larger the current is. As a side remark, we mention that for very low currents diffusion starts to play a role, which is also not covered by the model and thus leads again to wrong MEPP predictions for low voltage (at small $E_{c}$ ).

Also the arc attachment location $z_{A}$ of the torch example is kind of a "weak" mode. The system is axially translationally invariant in the anode region, and the anode column breaks this symmetry. This mode is not in conflict with any local conservation law. But it has a global effect via the dependence of the total voltage, or entropy production rate, on $z_{A}$ (it is thus not a zero-mode, in contrast to an azimuthal displacement of the anode spot). Again, if forces like magnetic force and drag force become strong, one has to expect that $z_{A}$ is determined by balancing them, and the MEPP result in its present form is no longer useful due to the inappropriateness of the constraints.

These experiences suggest the conjecture that the applicability of MEPP requires the presence of such a "weak" mode. Although, unfortunately, "weakness" is not clearly defined, it is correlated to the irrelevance of information. In other words, there are many weak, different physical effects of low relevance that would have to be taken into account in the missing equations for $\mathbf{z}$, and $\mathbf{z}$ is rather governed by entropic than energetic driving forces. If there would be a few strong physical forces that outbalance $\mathbf{z}$, one would know them. Or, according to Jaynes, if MEPP provides wrong results, one knows that relevant information was overlooked.

A different viewpoint on the interpretation of the occasional success of MEPP is based on work by Kohler [25], who has shown that for the linearized Boltzmann transport equation entropy production principles are valid. Again, both maximization and minimization principles hold - which one, depends on the type of constraints. In [26] it has been shown that radiation modeling in the framework of photo-hydrodynamics with an entropy production principle leads to reasonable results arbitrarily far from equilibrium. First, comparison of results suggest that this approach is superior to the often used entropy maximization approach. The reason is that entropy maximization pretends that the system is fully equilibrated on the constraint manifold, while if one considers entropy production rate, equilibration is explicitly taken into account. This is trivially more accurate under nonequilibrium conditions. Secondly, the fact that it works also far from equilibrium is believed to be related to the linearity of the underlying Boltzmann transport equation for the photon gas. As the term "near equilibrium" means "within the approximation of linear deviations from equilibrium", the considered photon gas is always "near equilibrium" on this kinetic level of description, whatever the photon distribution function looks like. Now the important point: despite of the linearity of the BTE, the resulting equations on the hydrodynamic level are generally strongly nonlinear. This characterizes the system far from equilibrium, even far from local equilibrium! Knowing only the photo-hydrodynamic equations, the underlying linearity is fully hidden. We will show elsewhere that the same can appear for electron transport in matter [27]. Another example, which points into this direction, is radiation induced material ablation [28]. There, MEPP was used to determine the vapor temperature and the ablation rate, parameters that enter into the boundary condition of the hydrodynamic balance equations for electric arc simulations. While global mass and energy balance was considered, momentum balance was not used because of local nonequilibrium in the evaporation (Knudsen) layer. The astonishingly good accordance of the MEPP result with experimental 
observations might also here be related to the behavior of the Knudsen layer to be well approximated by a linearized Boltzmann transport equation.

These observations suggest as a second conjecture, that good results obtained from MEPP for seemingly far from equilibrium systems might rely on a hidden linearity of the underlying Boltzmann transport equation for the concerned quantities.

\section{Acknowledgements}

I would like to thank Frank Kassubek for many critical comments and careful reading of the manuscript.

\section{References}

1. Steenbeck, M. Energetik der gasentladungen. Z. Phys. 1932, 33, 809.

2. Hoyaux, M.F. Arc Physics; Springer: Berlin Heidelberg, Germany, 1968.

3. Peters, T. Über den Zusammenhang des Steenbeckschen minimumprinzips mit dem thermodynamischen prinzip der minimalen entropieerzeugung. Z. Phys. 1956, 144, 612-631.

4. Although not fully correct in certain respects, we will generally identify Prigogine's principle with the principle of minimum entropy production rate.

5. Ichiyanagi, M. Variational principles of irreversible processes. Phys. Rep. 1994, 243, 125-182.

6. Martyushev, L.M.; Seleznev, V.D. Maximum entropy production principles in physics, chemistry and biology. Phys. Rep. 2006, 426, 1-45.

7. Non-equilibrium Thermodynamics and the Production of Entropy; Kleidon, A., Lorenz, R., Eds.; Springer: Berlin Heidelberg, Germany, 2005.

8. Christen, T. Application of the maximum entropy production principle to electrical systems. J. Phys. D: Appl. Phys. 2006, 39, 4497-4503.

9. Finkelnburg, W.; Maecker, H. Elektrische Bögen und Thermisches Plasma, Encyclopedia of Physics; Flügge, S., Ed.; Springer: Berlin, Germany, 1956; Vol. XXII, p. 254.

10. Christen, T.; Seeger, M. Simulation of unipolar space charge controlled electric fields. J. Electrostat. 2007, 65, 11-20.

11. Takahashi, T.; Hayakawa, N.; Yuasa, S.; Okabe, S.; Okubo, H. Space charge behaviour and corona stabilization effect in $S F_{6}$ gas viewed from sequential generation of a dc partial discharge. J. Phys. D: Appl Phys. 2001, 34, 1878-1884.

12. Lampert, M.A.; Mark, P. Current Injection in Solids; Academic Press: New York, NY, USA, 1970.

13. Paik, S.; Huang, P.C.; Heberlein J.; Pfender, E. Determination of the arc-root position in a DC plasma torch. Plasma Chem. Plasma Process. 1993, 13, 379-397.

14. Li, H.-P.; Pfender, E.; Chen, X. Application of Steenbeck's minimum principle for three-dimensional modelling of DC arc plasma torches. J. Phys. D: Appl Phys. 2003, 36, 1084-1096.

15. Bruers, S.; Maes, C.; Netocny, K. On the validity of entropy production principles for linear electric circuits. J. Stat. Phys. 2007, 129, 725-740. 
16. Landauer, R. Inadequacy of entropy and entropy derivatives in characterizing the steady state. Phys. Rev. A 1975, 12, 636-638.

17. Gage, D.H.; Schiffer, M.; Kline, S.J.; Reynolds, W.C. Nonequilibrium Thermodynamics, Variational Techniques, and Stability; Donnelly, R.J., Herman, R., Prigogine, I., Eds.; University of Chicago Press: Chicago, IL, USA, 1966; p. 283.

18. Grinstein, G.; Linsker, R. Comments on a derivation and application of the "maximum entropy production" principle. J. Phys. A: Math. Theor. 2007, 40, 9717-9720.

19. Benilov, M.S. Understanding and modelling plasma-electrode interaction in high-pressure arc discharges: a review. J. Phys. D: Appl Phys. 2008, 41, 144001-144030.

20. Jaynes, E.T. The minimum entropy production principle. Ann. Rev. Phys. Chem. 1980, 31, 579-601.

21. Jones, W. Variational principles for entropy production and predictive statistical mechanics. J. Phys. A: Math. Gen. 1983, 16, 3629-3634.

22. Rebhan, E. Maximum entropy production far from equilibrium: the example of strong shock waves. Phys. Rev. A 1990, 42, 781-788.

23. Seeger, M.; Niemeyer, L.; Christen, T.; Schwinne, M.; Dommerque, R. An integral arc model for ablation controlled arcs based on CFD simulations. J. Phys. D: Appl. Phys. 2006, 39, 2180-2191.

24. Christen, T.; Seeger, M. Current interruption limit and resistance of the self-similar electric arc. $J$. Appl. Phys. 2005, 97, 106108-106110.

25. Kohler, M. Behandlung von Nichtgleichgewichtsvorgngen mit Hilfe eines extremalprinzips. Phys. Zeitschr. 1948, 124, 772-789.

26. Christen, T.; Kassubek, F. Minimum entropy production closure of the photo-hydrodynamic equations for radiative heat transfer. J. Quant. Spec. Rad. Transf. 2009, 110, 452-463.

27. Christen, T. Steady state distribution function for a gas of independent electrons far from equilibrium. 2009, arXiv:0909.2774v2. arXiv.org e-Print archive. Available online:

http://arxiv.org/abs/0909.2774v2 (accessed December 8, 2009).

28. Christen, T. A maximum entropy production model for Teflon ablation by arc radiation. J. Phys. D: Appl. Phys. 2007, 40, 5719-5722.

\section{Appendix}

We give a simple example that shows graphically, that the difference between minimum and maximum entropy production rate principles is related to the convexity properties of the optimization problem. Consider in Figure 1 a resistance $R_{0}=0$ and a system $\Omega$ that consists of two constant resistors $R$ in parallel with unknown currents $z_{1}$ and $z_{2}$. We know that $I=z_{1}+z_{2}$ and $T_{a m b} \dot{S}=R\left(z_{1}^{2}+z_{2}^{2}\right)$. Power balance, as the constraint for the isolated system, reads $U\left(z_{1}+z_{2}\right)=R z_{1}^{2}+R z_{2}^{2}$, which gives a circle in the $z_{1}-z_{2}$-plane. Prigogine's principle, on the other hand, fixes the current, i.e., $I=z_{1}+z_{2}=$ constant, which is a straight line in this plane. The contour lines of $\dot{S}$, as well as the two constraints are plotted in Figure 5. The steady state is given by the point where the contour line has a point of contact with the constraint. Both cases reproduce the correct result. But along the constraint curves, $\dot{S}$ becomes minimum for constant $I$ (dotted curve) and maximum for constant $U$ (dashed curve). It is obvious, that Prigogine's principle is a convex optimization problem ( $\dot{S}$ is convex, and the constraint is affine), and thus leads 
to a minimum. One the other hand, the power balance constraint is different: its curvature is larger than the curvature of the $\dot{S}$-contour, hence the optimization problem is not convex. This illustrates, in our context, the mathematical difference between maximum and minimum entropy production rate principles.

Figure 5. Illustration of the type of optimum of the solution (black dot). Solid curves: contour lines of $\dot{S}$; dashed curve: power balance constraint; dotted curve: constant current constraint.

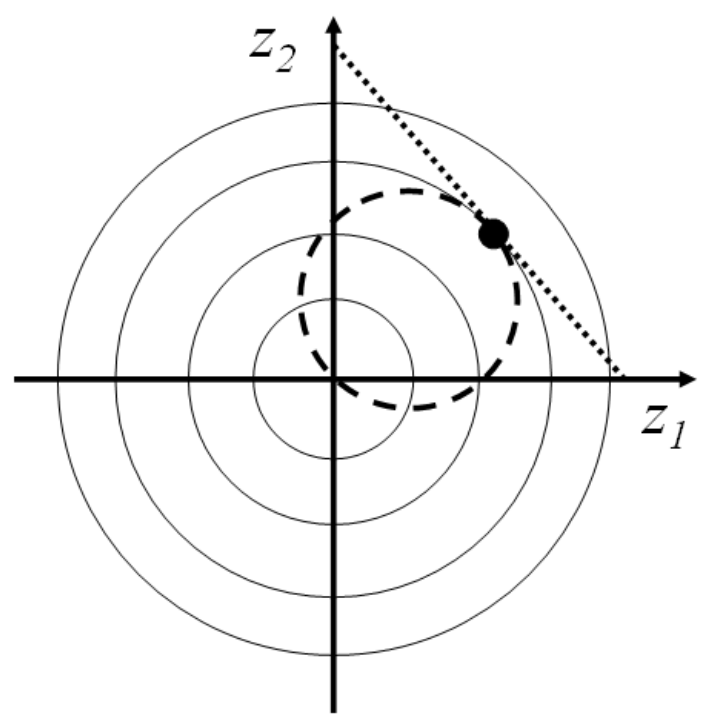

(c) 2009 by the author; licensee Molecular Diversity Preservation International, Basel, Switzerland. This article is an open-access article distributed under the terms and conditions of the Creative Commons Attribution license http://creativecommons.org/licenses/by/3.0/. 